\title{
Fluorescent Property of Carbon Nanodots Synthesized by Microwave Method from Crystal Nanocellulose as Precursor
}

\author{
Marpongahtun ${ }^{1}$, Saharman Gea ${ }^{2}$, Yugia Muis ${ }^{3}$, Andriayani ${ }^{4}$, Yolandha Putri ${ }^{5}$, Averroes F \\ Piliang $^{6}$ \\ \{marpongahtun@usu.ac.id ${ }^{1}$, s.gea@usu.ac.id ${ }^{2}$ \} \\ Universitas Sumatera Utara, Jalan Bioteknologi No.1, Medan 20155, Sumatera Utara, \\ Indonesia $^{1,2,3,4,5,6}$
}

\begin{abstract}
Carbon nanodots (CDots) are nanoparticles that can be synthesized from various carbon sources. In synthesizing the Dots by using carbon source from oil palm empty fruit as raw material, cellulose could become a potential source. Cellulose nanocrystals were utilized as precursors, and the Cdots were obtained by using microwave method. In this study, CDots synthesis using microwave method has been successfully done. From FTIR analysis, cellulose isolated from oil palm empty fruit showed functional groups which corresponded to neat cellulose. And for TEM analysis showed rod forms needles arranged variously, and they have diameter length about 10 , $9 \mathrm{~nm}$. The physical properties of Cdotswere obtained in the most optimum parameters during the conditions of 1000 watts for 20 minutes. The obtained results were dark brown colour with green-to-blue fluorescent under UV light source. From this study, it can be concluded that CDots can be synthesized from cellulose nanocrystals.
\end{abstract}

Keywords: Carbon Nanodots, Cellulose Nanocrystals, Fluorescent.

\section{Introduction}

Oil palm, Elaisguineensisis one of the most economical perennial oil crops that have 36 million tons (odmt) per year of fronds from pruning and replanting (Wan et al., 2004) and 5.2 million tons (odmt) per annum of empty-fruit bunches (EFB) in 2002 (Tanaka and Yamamoto, 2004). Each ton of CPO produces 1.1 ton of Oil Palm Empty- Fruit- Bunches (OPEFB) and causes serious adverse environmental impacts (Law et al., 2007; Sudiyani et al., 2013). OPEFB which consists of 44, 4\% cellulose, 30, 9\% hemicellulose and 14, 2\% lignin has big potential as raw material in synthesizing carbonaceous material. These three components can be converted into various chemical material and valuable products.

Cellulose nanocrystals are 100-250 nm long and 2-6 nm wide. Consist of highly crystalline nanosize segments is obtained from cellulose fibres when the amorphous regions are eliminated by acid hydrolysis (Eichhorn, 2011).However, in removing the lignin, mechanical and chemical properties are essentially needed. Strong acid like sulphuric acid, nitric acid and chloric acid have been used selectively to hydrolyse amorphous forms from cellulose (Tang et al., 2011), while the steam explosion supported with alkaline treatment have been successfully obtained in nano-scale (Gea et al., 2018b). 
Carbon nanodotsbelong to a new class of carbon-based nanomaterial with size less than $10 \mathrm{~nm}$ (Baker and Baker, 2010; Sahu et al., 2012). These materials were accidentally discovered during the electrophoretic purification of single-walled carbon nanotubes (SWCNTs) in 2004 (Xu et al., 2004). Carbon nanodots have outstanding water solubility, high sensitivity and selectivity to target analytes, low toxicity, favourable biocompatibility, and excellent photo stability, and their existence is abundant in nature (Li et al., 2012). Nowadays, carbon nanodots have been attracted researcher widely because of their strong fluorescence emission in the visible and near-infrared (Bourlinos et al., 2008; Cao et al., 2007). With their excellent properties, carbon nanodots still develop rapidly these days.

Like synthesizing carbon nanotubes (CNT) by using temperature process, the carbon nanodots are obtained with similar process. It has been reported that the coffee waste was used to synthesize carbon nanodotswith high temperature over $500^{\circ} \mathrm{C}$ with 12 hour of oxidation process (Gea et al., 2018a). Carbon chain bond as main source for fabrication of carbon nanodots becomes focus experimental that has been researched in recent years. Various improvements had been achieved about the study of carbon nanodots synthesis or properties, and applications have been reported by researchers.

Therefore, this study is conducted to investigate the effect of time temperature in microwave to reduce the high consumption of energy.

\section{Materials And Methods}

\subsection{Materials}

Oil Palm Empty Fruit Bunches (OPFEB), distilled water, and dialysis membrane, nitric acid, sulphate acid, sodium hydroxide, sodium hypochlorite $12 \%$, acetate acid.

\subsection{Nanocrystals Cellulose Isolation}

\subsubsection{Alkaline Treatment}

As many as $50 \mathrm{~g}$ powders of OPEFB were put in beaker glass; the sieved fibres were dispersed in distilled water. Then the suspension was stirred for 2 hours at $50 \mathrm{oC}$ and filtered, and this procedure was repeated once more. The residue was dispersed in a $100 \mathrm{ml}$ of $2 \% \mathrm{NaOH}$ solution and the suspension was stirred for 2 hours at $80 \mathrm{oC}$, filtered and washed with water. This alkaline treatment was repeated once more and the fibres were dried at $50 \mathrm{oC}$ for 24 hours.

\subsubsection{Bleaching Treatment}

This process is also referred as "Delignification process". The alkali pretreated fibers were undergone bleaching treatment using sodium chlorite solution and 8 to 10 drops of glacial acetic acid while being heated at $60^{\circ} \mathrm{C}$ to $70^{\circ} \mathrm{C}$ so that the lignin content was removed in this process. The mixture was stirred at frequent intervals for 1 hour, cooled in ice bath, filtered quantitatively and washed with cold water. At the end, the bleached pulps were treated with $0.05 \mathrm{~N}$ nitric acid solutions for 1 hour at $70^{\circ} \mathrm{C}$ and washed extensively with water. The pulps were heated for 1 hour by using $\mathrm{NaOH} 17,5 \%$, and then they were filtered and washed until 
the $\mathrm{pH}$ neutral. Bleaching treatment using $\mathrm{NaOCl} 5 \%$ were applied and they were dried to produce alpha cellulose. FTIR was used to analyze cellulose.

\subsubsection{Preparation of Nanocrystals}

As many as $1 \mathrm{~g}$ cellulose hydrolysis was carried out using $45(\mathrm{w} / \mathrm{w})$ concentrated sulphate acid adding drop by drop until desired concentration is reached. The acid treated fibres were stirred constantly using mechanical stirrer, heating it simultaneously for 45 minutes. In these processes the hydrogen bonds break forming complexes and sulphate esters, depolymerization of macromolecular chains, finally change in crystallinity. The suspension was centrifuged at $10000 \mathrm{rpm}$ for 15 minutes and ultra-sonicated for well dispersion of nanomaterial preventing agglomeration. Then, inserted into dialysis membrane then stirred for 4 days in $100 \mathrm{ml}$ of distilled water then evaporated at $70 \mathrm{oC}$ to produce nanocrystal cellulose. TEM was used to analyse nanocrystal cellulose.

\subsubsection{Preparation of Carbon Nanodots}

The microwave treatment was applied to produce C-Dots. As many as $200 \mathrm{mg}$ nanocrystal cellulose was placed into microwave with various times i.e. 10 minutes, 15 minutes, and 20 minutes. After being treated, the samples were performed under UV light and they were placed under UV light to investigate the fluorescence properties.

\subsection{Characterization}

\subsubsection{Characterization with FTIR Spectroscopy}

The samples were placed on plate to direction of infrared rays then clamped.The results were obtained infrared absorption peaks from samples in wave number and transmittance plot on the monitor with range of wave number $4000-5000 \mathrm{~cm}^{-1}$.

\subsubsection{Characterizationwith TEM}

The samples were dripped by ammonium molibdate $2 \%$, and then the solution was trapped in resin. Forward, cut with micro cutting to obtain single nanocrystals. Cellulose morphology analysis was carried out using a JEOL 1400 with an acceleration voltage of $120 \mathrm{kV}$.

\subsubsection{Characterization with UV light}

The samples were dissolved with distilled water and then put under UV light and observed fluorescent formed. 


\section{Result And Discussion}

\subsection{FTIR Studies}

FTIR studies confirmed the successful isolation of alpha cellulose from Oil Palm Empty Fruit Bunches (OPEFB) and displayed in Figure 1. The peak at $3363,86 \mathrm{~cm}^{-1}$ showed vibration of $\mathrm{O}-\mathrm{H}$ - from alpha cellulose. The absorption band at the wave number $2900,94 \mathrm{~cm}^{-1}$ is the C-H group, $1620 ; 21 \mathrm{~cm}$ is the $-\mathrm{C}-\mathrm{O}$ - group. Then, wave number of $1319,31 \mathrm{~cm}^{-1}$ which is a stretching C-O-C group and 1056,99 is the -C-O- group, vibration of 894,97 showed of $-\mathrm{C}-\mathrm{H}-$ group.From the results obtained can be said that alpha cellulose isolated from oil palm empty fruit has been successfully carried out. Based on specific groups for cellulose are $-\mathrm{OH},-\mathrm{CH}_{2}$,O-, which repeatedly appears at FT-IR spectrum, and the $-\mathrm{OH}$ at $3363,66 \mathrm{~cm}^{-1}$ are stretching vibration of $-\mathrm{OH}$.

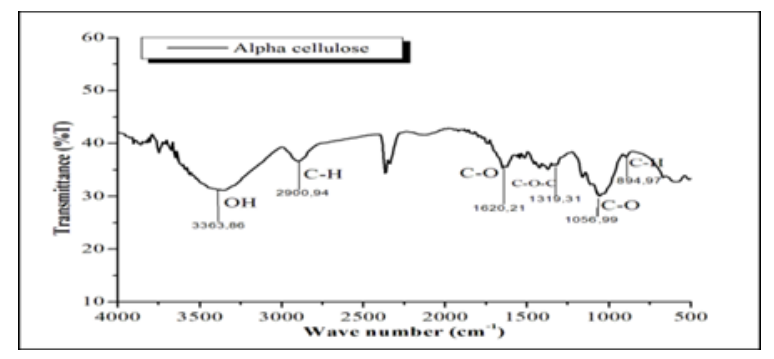

Fig. 1.FTIR alpha cellulose.

\subsection{Nanocrystal Morphology}

TEM analysis was used to determine surface morphology and can also enlarge objects in small sizes. From TEM results obtained in cellulose nanocrystals is displayed in Figure 2, where rod-like forms from cellulose nanocrystals were arranged variously, like needles.

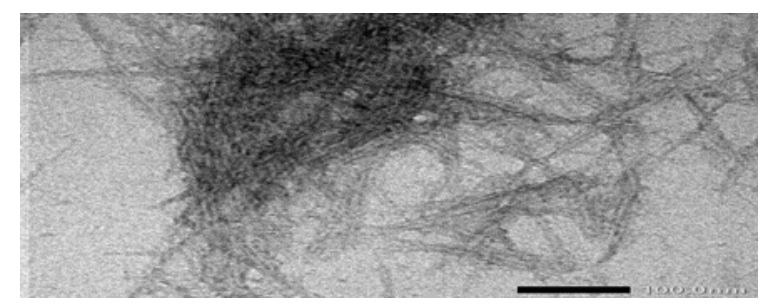

Fig. 2.TEM Nanocrystal

Cellulose nanocrystals are cellulose produced from nanotechnology which is a dimensional material from 0, 2-100 nm. From TEM results obtained can be known the diameter size of cellulose nanocrystals with ImageJ application. Based on calculations performed, obtained the diameter size of cellulose nanocrystals range between $10,9 \mathrm{~nm}$. With particle size distribution shown in Figure 3. 


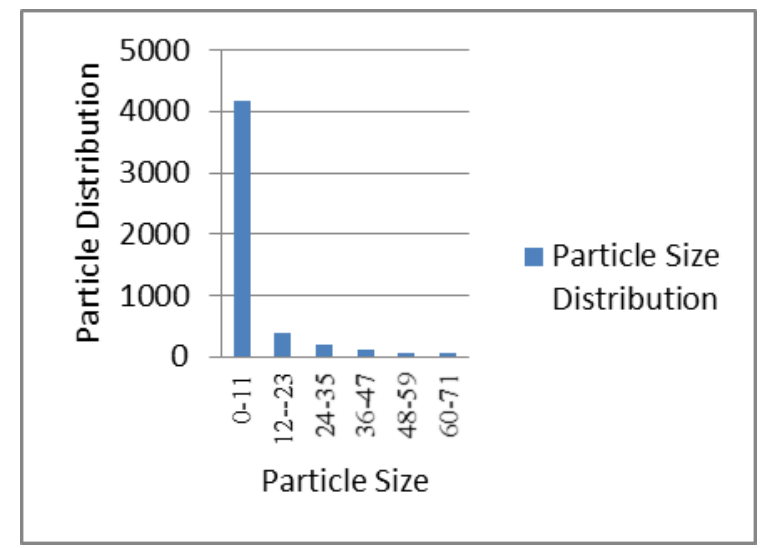

Fig. 3. Particle Size Distribution

The result that was obtained provided that time affects the fluorescence of C-dots. Heating for 20 minutes showed greater result than othersand displayed in figure 4 . Heating for 20 minutes showed the fluorescence of C-dots under visible light showed colourless transparent and under UV light showed green- to- bluefluorescence. This shows that CDots can be synthesized from cellulose nanocrystals with fluorescent on them.

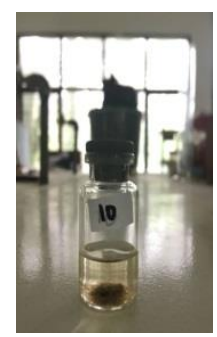

(a)

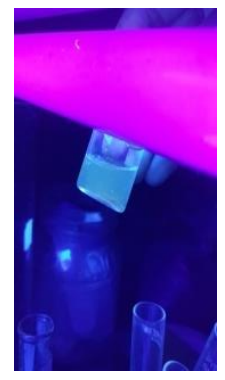

(d)

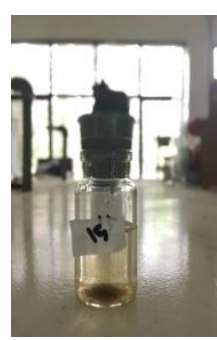

(b)

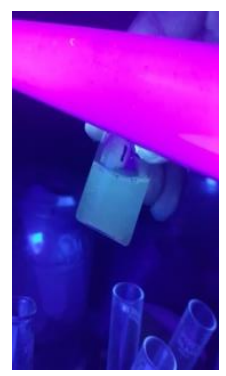

(e)

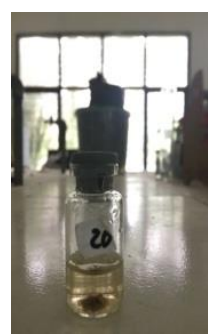

(c)

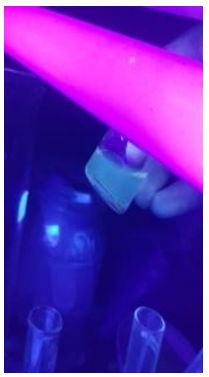

(f)

Fig. 4.Fluorescence images under visible light and under UV light (a) The samples heated with 20 minutes (b) The samples heated with 15 minutes (c) with 10 minutes. While the (d), (e), and (f), are the fluorescent images of previous samples respectively 


\section{Conclusion}

The experimental results of C-Dots showed that nanocrystal cellulose as precursor produced from empty bunch of palm oil were able to be synthesized by using microwave treatment. Based on microwave treatment at 20 minutes heating showed the green fluorescence. Microwave heating provides a fast, reliable, and high-yield procedure for producing fluorescent carbon nanoparticles facilitating research on various synthetic schemes based on single or precursor material.

Acknowledgement.Acknowledgements are given to Universitas Sumatera Utara and Ministry of Research, Technology and Higher Education for its research grants throughout program PDUPT 2018 with given contract No. 2590/UN5.1.R/PPM/2018.

\section{References}

[1]Baker, S.N., Baker, G.A., 2010. Luminescent carbon nanodots: Emergent nanolights. Angew. Chemie - Int. Ed. https://doi.org/10.1002/anie.200906623

[2]Bourlinos, A.B., Stassinopoulos, A., Anglos, D., Zboril, R., Georgakilas, V., Giannelis, E.P., 2008. Photoluminescent carbogenic dots. Chem. Mater. https://doi.org/10.1021/cm800506r

[3]Cao, L., Wang, X., Meziani, M.J., Lu, F., Wang, H., Luo, P.G., Lin, Y., Harruff, B.A., Veca, L.M., Murray, D., Xie, S.Y., Sun, Y.P., 2007. Carbon dots for multiphoton bioimaging. J. Am. Chem. Soc. https://doi.org/10.1021/ja0735271

[4]Eichhorn, S.J., 2011. Cellulose nanowhiskers: Promising materials for advanced applications. Soft Matter. https://doi.org/10.1039/c0sm00142b

[5]Gea, S., Tjandra, S., Joshua, J., Wirjosentono, B., 2018a. Morphological study of fluorescent carbon Nanoparticles (F-CNPs) from ground coffee waste soot oxidation by diluted acid, in: IOP Conference Series: Materials Science and Engineering. https://doi.org/10.1088/1757-899X/309/1/012037

[6]Gea, S., Zulfahmi, Z., Yunus, D., Andriayani, A., Hutapea, Y.A., 2018b. The Isolation of Nanofibre Cellulose from Oil Palm Empty Fruit Bunch Via Steam Explosion and Hydrolysis with $\mathrm{HCl} \mathrm{10 \% ,} \mathrm{in:}$ Journal of Physics: Conference Series. https://doi.org/10.1088/1742-6596/979/1/012063

[7]Law, K.N., Daud, W.R.W., Ghazali, A., 2007. Morphological and chemical nature of fiber strands of oil palm empty-fruit-bunch (OPEFB). BioResources. https://doi.org/10.15376/biores.2.3.351-362

[8]Li, H., Kang, Z., Liu, Y., Lee, S.T., 2012. Carbon nanodots: Synthesis, properties and applications. J. Mater. Chem. https://doi.org/10.1039/c2jm34690g

[9]Sahu, S., Behera, B., Maiti, T.K., Mohapatra, S., 2012. Simple one-step synthesis of highly luminescent carbon dots from orange juice: Application as excellent bio-imaging agents. Chem. Commun. https://doi.org/10.1039/c2cc33796g

[10]Sudiyani, Y., Styarini, D., Triwahyuni, E., Sudiyarmanto, Sembiring, K.C., Aristiawan, Y., Abimanyu, H., Han, M.H., 2013. Utilization of biomass waste empty fruit bunch fiber of palm oil for bioethanol production using pilot - Scale unit, in: Energy Procedia. https://doi.org/10.1016/j.egypro.2013.05.005

[11]Tanaka, R., Yamamoto, K., 2004. Accumulation and Availability of Oil Palm Biomass in Malaysia. JIRCAS Work. Rep. 39, 20-34.

[12]Tang, L. rong, Huang, B., Ou, W., Chen, X. rong, Chen, Y. dan, 2011. Manufacture of cellulose nanocrystals by cation exchange resin-catalyzed hydrolysis of cellulose. Bioresour. Technol. https://doi.org/10.1016/j.biortech.2011.09.070

[13]Wan, Z., Sato, M., Furuich, J., Sukri, S., Sukri, M., 2004. Recent Development on The Processing and Utilization of Complete Feed Based on Oil Palm Fronds for Ruminant Feeding in Malaysia. JIRCAS Work. Rep. 39, 24-27. 
[14]Xu, X., Ray, R., Gu, Y., Ploehn, H.J., Gearheart, L., Raker, K., Scrivens, W.A., 2004. Electrophoretic Analysis and Purification of Fluorescent Single-Walled Carbon Nanotube Fragments. J. Am. Chem. Soc. https://doi.org/10.1021/ja040082h 